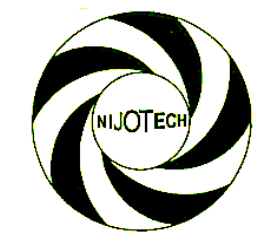

Nigerian Journal of Technology (NIJOTECH)

Vol. 39, No. 2, April 2020, pp. 514 - 527

Copyright@ Faculty of Engineering, University of Nigeria, Nsukka

Print ISSN: 0331-8443, Electronic ISSN: 2467-8821

www.nijotech.com

http://dx.doi.org/10.4314/njt.v39i2.22

\title{
INTELLIGENT LEARNING DIVERSITY MECHANISM FOR UNMANNED AERIAL VEHICLES APPLICATIONS
}

\author{
A. A. Periola ${ }^{1, *}$ and E. Obayiuwana ${ }^{2}$

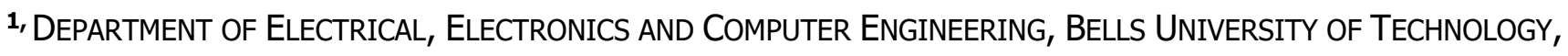 \\ OTTA, OGUN STATE, NIGERIA \\ 2, Department of Electrical and Electronics Engineering, Obafemi AWolowo University, Ile-Ife, Osun \\ STATE, NIGERIA
}

E-mail addresses: 1 periola@hotmail.com, ${ }^{2}$ write2uwa@yahoo.co.uk

\begin{abstract}
The increased use of drones and aerial vehicles in applications poses challenges of airspace safety for aviation organizations. It is important to ensure the safety of the airspace when a significant number of unmanned aerial vehicles are deployed by civilian users. $A$ solution that meets this requirement is important to promote innovation in the commercialization of air space for civilian users deploying unmanned aerial vehicle. The discussion in this paper proposes a mechanism that uses artificial intelligence to address this challenge. The proposed mechanism utilizes a low altitude platform (LAP) and entities in terrestrial wireless networks. The low altitude platform (LAP) observes, develops insights and training data (with human aid). The training data is used to develop learning mechanisms which determine the suitable unmanned aerial vehicles flight parameters in different scenarios. The use of the LAP reduces the burden of communicating with terrestrial base stations. The unmanned aerial vehicles have a reduced altitude between the LAPs in comparison to terrestrial base stations. This reduces the free space path loss and rain-induced attenuation. The performance benefit of the proposed mechanism in comparison to existing solution is examined via MATLAB simulations. Evaluation shows that the proposed mechanism reduces the network access costs by up to $90 \%$ on average. The proposed mechanism also increases available flight power and improves airspace safety by $37.3 \%$ and up to $53.2 \%$ on average respectively.
\end{abstract}

Keywords: Autonomous unmanned aerial vehicles, Intelligence Paradigm; Aviation Safety, Capital Constrained Aviation Organizations.

\section{INTRODUCTION}

Advances in aerial vehicle technology have led to the emergence of numerous applications in areas such as near space communications [1-3], weather monitoring [4-6] and scientific studies [7-9]. The use of aerial vehicles is attractive due to their low cost benefit in comparison to satellites. However, the large scale use of aerial vehicles faces challenges due to aviation safety risks [10-12]. It is important to design solutions that address the safety concern associated with the use of aerial vehicles. This enables more capital constrained aviation organizations to deliver new applications via aerial vehicles. Capital constrained aviation organizations are those that cannot afford manned aerial vehicles and pilots in the long term. The challenge addressed in this paper is ensuring that capital-constrained aviation organizations can deploy unmanned aerial vehicles for service delivery (across different applications) while meeting aviation safety requirements. This paper makes the following contributions:

Firstly, the paper proposes a novel mechanism enabling unmanned aerial vehicles to meet aviation safety requirements using artificial intelligence. The proposed mechanism makes use of a low altitude

* Corresponding author, tel: +234-809-479-6001 
platform (LAP) that incorporates learning diversity. The LAP incorporates payload enabling it to observe bird behaviour, and develop insights that can be used to derive training data with human assistance from a ground location. The derived training data is used to train learning algorithms aboard the LAP. These learning algorithms are transferred to the unmanned aerial vehicles for autonomous guidance. Each unmanned aerial vehicle uses a different learning algorithm for motion guidance in different contexts. Unmanned aerial vehicle obtains information on learning mechanism suitability from the LAP.

Secondly, the paper formulates the performance model for the proposed mechanism. The formulated metrics are (i) network access costs, (ii) available flight power on unmanned aerial vehicles before and after using the proposed mechanism, and (iii) the aerial aviation safety indicator (AASI) before and after using the proposed mechanism. The AASI metric investigates how artificial intelligence paradigms enhance aerial safety.

The rest of the paper is organized as follows. Section 2 discusses the motivation and related background to the research described in the paper. Section 3 describes the problem. Section 4 presents the proposed mechanism. Section 5 formulates the performance model. Section 6 presents and discusses simulation results. Section 7 concludes the paper.

\section{MOTIVATION AND RELATED BACKGROUND}

The discussion here examines the existing use of aerial vehicles in different applications. Aerial vehicles can be considered as either manned aerial vehicles or unmanned aerial vehicles [13-15]. The use of unmanned aerial vehicles poses aviation safety risks since there is no human on-board. Unmanned aerial vehicles are controlled via signals sent from a ground station. Manned aerial vehicles have a human onboard and pose a lesser safety risk than unmanned aerial vehicles. Hence, the use of manned aerial vehicles is more favourable to airspace regulatory agencies from the viewpoint of ensuring safety.

This perspective has motivated Ogan [16] to propose the joint use of manned aerial vehicles and unmanned aerial vehicles. The use of manned aerial vehicles is proposed to mitigate against the safety risks caused by increased use of unmanned aerial vehicles. However, the use of manned aerial vehicles incurs higher costs than autonomous unmanned aerial vehicles. This is because operators of manned aerial vehicles have to hire and train pilots responsible for operating the manned aerial vehicle. However, pilots are not required for autonomous unmanned aerial vehicles. The additional cost of hiring and training of pilots makes the large scale use of piloted manned aerial vehicles expensive for capital-constrained aviation organizations. In a case where pilots have high fees, the cost of hiring a significant number of pilots can exceed the cost of acquiring manned aerial vehicles. This is not beneficial for capital constrained aviation organizations.

An unmanned aerial vehicle can be remotely piloted or engage in autonomous flight guidance [16]. A remotely piloted unmanned aerial vehicle receives flight guidance signal via wireless network connection. The wireless network connection is also used to resolve aerial traffic alert concerns. The use of remotely piloted unmanned aerial vehicles poses challenges to terrestrial wireless networks. For example, the large scale deploying of remotely piloted unmanned aerial vehicles increase the risk of congestion on terrestrial wireless networks. The congestion arises when a significant number of remotely piloted unmanned aerial vehicles are in a given coverage area. The relay of control signals for remotely piloted unmanned aerial vehicles is also subject to the state of terrestrial wireless networks. Control signal transmission suffers a high latency when terrestrial wireless networks have a poor quality of service. This increases safety risks.

In addition, capital constrained aviation organizations should be able to pay wireless network operators where necessary for network access. This is because the providing network access is a revenue source for wireless network operators. The network access charges for transmitting control signals to remotely piloted unmanned aerial vehicles become very high when multiple aerial vehicles are deployed.

The use of autonomous unmanned aerial vehicles reduces the need to pay high network access charges and reduces the susceptibility of control signals to network latency effects. These benefits arise because autonomous unmanned aerial vehicles rely on intelligent algorithms for flight guidance.

The deep learning algorithm is suitable for designing intelligent behaviour for unmanned aerial vehicle [17]. Carrio et al. [17] recognize that deep learning algorithms are suitable for planning and situational awareness in unmanned aerial vehicle flight planning. Deep learning neural network algorithms are suitable for the execution of flight planning in dynamic aerial 
environments. Therefore, autonomous unmanned aerial vehicles should be equipped with sensor payload enabling them to acquire the environmental state. The acquired environmental state is used to determine the output of the deep learning algorithm. The output determines the action executed by the autonomous unmanned aerial vehicle. The discussion in [17] also recognizes challenges affecting achieving autonomy in unmanned aerial vehicles. Some of these challenges are size, weight and payload power restrictions. The realization of feature extraction to realize deep learning in unmanned aerial vehicles is also challenging. For example, the size restriction of unmanned aerial vehicle limits the computing payload that can be integrated on autonomous unmanned aerial vehicle.

In [18], Kouris et al. propose the use of convolutional neural networks to solve the problem of autonomous unmanned aerial vehicle navigation. The unmanned aerial vehicle incorporates ultrasonic sensors for long range distance sensing. The authors in [18] aim to create an indoor flight dataset annotated with real distance labels. The acquisition of training data is realized by mounting three pairs of Ultrasonic and Infra-Red distance sensors on the unmanned aerial vehicle. The developed algorithm targets indoor environments and not outdoor environment.

The development of landing hardware for unmanned aerial vehicles is addressed in [19] by Luo et al. The discussion in [19] is motivated by the observation that flying animals utilize soft landing ability enabling them to land on different types of surfaces. The paper i.e. [19] aims to equip unmanned aerial vehicles with this landing ability. The bioinspired technique utilized radial basis function neural network proportional integral derivative controllers. The proposed technique has the benefit of enabling the Quadcopter drone being considered to land on a non-flat surface. The ability to land on non-flat surfaces enhances safety because unmanned aerial vehicles can take advantage of more surfaces to ensure safety.

Luo et al. [19] considers the usefulness of the landing ability of birds in improving unmanned aerial vehicles landing ability. However, birds have more useful behaviour that can be considered in designing unmanned aerial vehicles with improved safety capabilities. For example, birds also make use of the airspace and are equipped with evolving mechanisms to ensure airspace safety.
Lyons et al. in [13] examine the response of birds to drones. They observe that bird behaviour do not pose a significant threat to small unmanned aerial vehicles i.e. drones. The response of birds to drones sharing their territory is observed to have unexpected safety consequences in some instances. However, the incorporation of drone manoeuvrability is capable of evading negative responses for birds with territorial behaviour. The discussion in [13] is aimed at studying how drone deployment influences bird behaviour in breeding and non-breeding seasons. This study has biological significance and aims to ensure that drone use does not reduce avian population.

Flight is a challenging task requiring significant muscular activity in birds. In addition, successful bird flights require cognitive capability. This is because birds respond to different threat scenarios during flight. The muscle activity is also influenced by avian neuron activity [20]. Therefore, unmanned aerial vehicle flight behavior can be influenced by mechanisms motivated by avian neuron activity. Research has focused on developing unmanned aerial vehicle flight behaviour [19, 21-25] but lesser research has focused on developing neuronal networks motivated by avian intelligence. This is because of lesser understanding on the role of cognitive ornithology in bird flight and associated adaptation.

Efforts to incorporate bird like structures in unmanned aerial vehicles to enhance their flight performance should be matched with corresponding development in control logic. This control logic can be realized via artificial intelligence mechanisms such as artificial neural networks [17-18].

Artificial neural networks are suitable for designing robust and adaptive systems [26]. The concept of learning diversity is presented in [26]. Learning diversity implies the use of multiple learning algorithms to achieve a given goal at different epochs. The suitability of learning diversity is examined for cognitive radios in [26]. The use of multiple learning algorithms is beneficial because different learning algorithms are suitable for executing prediction in different scenarios with varying prediction accuracy. However, the use of learning diversity has not been considered in autonomous unmanned aerial vehicles.

Autonomous unmanned aerial vehicles are beneficial for capital constrained aviation organizations. This is because their use does not require having access to pilots or wireless network 
links (ideally avoiding network access costs). Currently, drone control is considered to require having access to terrestrial wireless networks access links [27-28]. This does not consider the widespread use of autonomous unmanned aerial vehicles and the interest of capital constrained aviation organizations. Moreover, autonomous unmanned aerial vehicles utilizing artificial intelligence mechanism require access to training data. Hence, autonomous unmanned aerial vehicles need wireless networks access for training data transfer.

Capital constrained aviation organizations deploying unmanned aerial vehicles in large numbers without access to wireless network links need to be able to adapt to changing flight scenarios. The resulting challenge can be addressed if newly developed intelligent mechanisms are transmitted to deployed autonomous unmanned aerial vehicles. The training data used to develop these new mechanisms is acquired by unmanned aerial vehicles (with limited computing payload) and transmitted to computing entities. This should be done while minimizing network access costs. Drones should also be able to adapt to new challenging scenarios emerging in the airspace. This should be achievable for capital constrained aviation organizations without recourse to terrestrial wireless networks. This paper proposes a solution that achieves this objective.

\section{PROBLEM DESCRIPTION}

This section describes the problem being addressed in this paper. The scenario being considered is one in which different capital constrained aviation organizations deploy multiple autonomous unmanned aerial vehicles in class $\mathrm{G}$ airspace. The autonomous aerial vehicles are used by capital constrained aviation organizations for service delivery to end-users. The set of capital constrained aviation organizations is denoted as $\alpha$ such that:

$$
\alpha=\left\{\alpha_{1}, \alpha_{2}, \ldots, \alpha_{I}\right\}
$$

Where $I$ is total number of capital constrained aviation organizations.

A capital constrained aviation organization can use multiple autonomous unmanned aerial vehicles. The $i^{\text {th }}$ capital constrained aviation organization $\alpha_{i}, \alpha_{i} \in \alpha$ can deploy multiple autonomous unmanned aerial vehicles. Let $\beta_{i}$ denote the set of autonomous unmanned aerial vehicles used by the $i^{\text {th }}$ capital constrained aviation organization $\alpha_{i}$.

$$
\beta_{i}=\left\{\beta_{i}^{1}, \beta_{i}^{2}, \ldots, \beta_{i}^{J}\right\}
$$

Where $J$ is the total number of autonomous unmanned aerial vehicle used by the $i^{\text {th }}$ capital constrained aviation organization $\alpha_{i}$.

In (2), $\beta_{i}^{2}$ is the second autonomous unmanned aerial vehicle used by the $i^{\text {th }}$ capital constrained aviation organization $\alpha_{i}$. The $j^{\text {th }}$ autonomous unmanned aerial vehicle used by the $i^{\text {th }}$ capital constrained aviation organization $\alpha_{i}$ is denoted $\beta_{i}^{j}, \beta_{i}^{j} \in \beta_{i}$.

The size of control data associated with the flight control of the autonomous unmanned aerial vehicle $\beta_{i}^{j}$ at epoch $t_{y}$ is denoted as $C_{d}^{1}\left(\beta_{i}^{j}, t_{y}\right)$. In addition, the size of the training data acquired by the concerned unmanned aerial vehicle sent to the ground segment for processing is denoted $C_{d}^{2}\left(\beta_{i}^{j}, t_{y}\right)$. The size of the training data $C_{d}^{2}\left(\beta_{i}^{j}, t_{y}\right)$ is unrelated to $C_{d}^{1}\left(\beta_{i}^{j}, t_{y}\right)$ i.e. $C_{d}^{1}\left(\beta_{i}^{j}, t_{y}\right) \neq C_{d}^{2}\left(\beta_{i}^{j}, t_{y}\right)$.

In addition, the cost of accessing wireless network links by the autonomous unmanned aerial vehicle $\beta_{i}^{j}$ at the $y^{\text {th }}$ epoch, $t_{y}$ is denoted $P_{a c}\left(\beta_{i}^{j}, t_{y}\right)$. A variable network access charge is feasible if the network incorporates congestion based dynamic pricing scheme. Given that the organization, $\beta_{i}^{j}$ has a threshold network access cost of the $i^{\text {th }}$ capitalconstrained aviation organization $P_{t h}\left(\alpha_{i}\right)$; the data transmission costs constitutes a bottleneck if:

$$
\begin{gathered}
\sum_{y=1}^{Y} \sum_{j=1}^{J} P_{a c}\left(\beta_{i}^{j}, t_{y}\right)\left(C_{d}^{1}\left(\beta_{i}^{j}, t_{y}\right)+C_{d}^{2}\left(\beta_{i}^{j}, t_{y}\right)\right) \\
\geq P_{t h}\left(\alpha_{i}\right)
\end{gathered}
$$

Furthermore, let $P$ and $P_{1}\left(\beta_{i}^{j}, t_{y}\right)$ denote the initial power aboard the autonomous aerial vehicle and power expended by the autonomous unmanned aerial vehicle $\beta_{i}^{j}$ at epoch $t_{y}$ respectively. Given that $P_{1}\left(\beta_{i}^{j}, t_{y}\right)$ also includes power consumption of the computing payload. The necessity of data communications reduces unmanned aerial vehicle flight duration if:

$$
P-\sum_{y=1}^{Y} P_{1}\left(\beta_{i}^{j}, t_{y}\right) \leq 0
$$

The aerial environment of the class $\mathrm{G}$ airspace comprises aerial vehicles from different capital constrained organizations and those of other aviation organizations. The other aviation organizations are those with access to significant financial, capital and technical resources such as Amazon. Let $\gamma$ be the set of other organizations with aerial vehicles in the class $\mathrm{G}$ airspace. 


$$
\gamma=\left\{\gamma_{1}, \gamma_{2}, \ldots, \gamma_{K}\right\}
$$

Where $K$ is the total number of additional aviation organizations in the class $\mathrm{G}$ airspace.

The set of autonomous aerial vehicles owned by organization $\gamma_{k}, \gamma_{k} \in \gamma$ is given as:

$$
\gamma_{k}=\left\{\gamma_{k}^{1}, \gamma_{k}^{2}, \ldots, \gamma_{k}^{L}\right\}
$$

Where $L$ is the total number of aerial vehicles used by the $k^{\text {th }}$ aviation organization.

The aerial environment of the autonomous unmanned aerial vehicle $\beta_{i}^{j}$ can comprise autonomous unmanned aerial vehicles from other aviation organizations. This is in addition to birds in the class $\mathrm{G}$ airspace. The vehicle $\beta_{i}^{j}$ needs to be capable of adapting for a safe flight in each possible class $\mathrm{G}$ airspace scenario. If the vehicle $\beta_{i}^{j}$ uses artificial neural networks for flight adaptation, it is feasible to think that an artificial neural network most suitable for a class $\mathrm{G}$ airspace scenario is unsuitable for another scenario.

This consideration is important as the scenario in the class $\mathrm{G}$ airspace is dynamic. This is because of the differences in the required training data and prediction accuracy for each scenario. Furthermore, different artificial neural networks have varying prediction accuracies making them capable of yielding different results in different scenarios. Autonomous unmanned aerial vehicles should also be able to benefit from newly emerging training data. A solution in this context requires a novel learning framework for autonomous unmanned aerial vehicle. This framework should also be supported by suitable computational payload. The computing payload should consider the power constraints of autonomous unmanned aerial vehicle in addressing this challenge.

Therefore, new mechanisms are required for capital constrained aviation organizations utilizing autonomous unmanned aerial vehicles in large-scale. The mechanism should address the challenges of (i) reducing wireless network link access costs, (ii) increasing power available for flight, (iii) enabling use of different machine learning algorithms at different epochs and (iv) improving airspace safety. The machine learning algorithms are developed using dynamic training data to improve adaptation and enhance safety.

\section{PROPOSED SOLUTION}

This section presents the proposed solution and is divided into three aspects. The first aspect presents the mechanism which enables the reduction of wireless network link access costs for autonomous unmanned aerial vehicles. The second aspect describes the solution enabling the improvement of available flight power in autonomous unmanned aerial vehicles. The third aspect presents a mechanism incorporating the concept of machine learning diversity.

\subsection{Proposed Solution - Reducing Wireless Network Link Access Costs}

Currently unmanned aerial vehicles obtain the control signals from terrestrial wireless networks [27-28]. The use of terrestrial wireless networks is advantageous in comparison to satellite networks due to the high cost of satellite links. Moreover, the higher distance between satellite networks and aerial vehicles signifies a high latency in comparison to terrestrial wireless networks with a shorter distance. The use of low altitude platforms (LAPs) is preferred in this case because they have a lower latency than satellite networks. LAPs also have a wide coverage, are located at an altitude above commercial aircraft and transmit signals to multiple unmanned aerial vehicles within their coverage.

This paper proposes a LAP crowd-sourcing acquisition strategy. In the proposed strategy, capital constrained aviation organizations jointly acquire or develop (via open source strategy) own LAPs. Recent advances favor an open source development of LAPs as seen in [29-30]. Multiple capital constrained aviation organizations can share the costs associated with acquiring or developing LAPs.

The LAPs receive signals from a ground location. The ground location is jointly acquired or developed by capital constrained aviation organizations. Data transmission from the ground location to the LAP; and from the LAP to the autonomous unmanned aerial vehicles is free of network access costs. This mechanism also reduces the risk of network congestion from the transmission of subscriber traffic in terrestrial wireless networks. The data transmitted in the proposed mechanism relates to ensuring the safety of autonomous unmanned aerial vehicle in the class $\mathrm{G}$ airspace. Transmitted data in this context comprises (i) acquired training data, (ii) preprocessed training data, (iii) matrices for developed artificial neural networks for predicting autonomous unmanned aerial vehicle manoeuvrability configuration parameters and (iv) sojourn status of the autonomous unmanned aerial vehicle. 
The observed information obtained via cameras and sensors on autonomous unmanned aerial vehicles is refined to extract suitable training data. This process can take place on either the LAP via remote terrestrial access and using the LAP's computing resources. It can also take place on the ground location after transmission of observed information.

The pre-processed training data is used to train artificial neural networks. This training can be done either on the LAP (with or without remote access) or at the ground station. In addition, the matrices for developed artificial neural networks are sent from either the ground station or the low altitude platform to the autonomous unmanned aerial vehicle in the class $\mathrm{G}$ airspace.

In the proposed mechanism, the LAP hosts information on the matrices of different learning algorithms suitable for use by autonomous aerial vehicles in ensuring airspace safety. This shifts the computational load from autonomous unmanned aerial vehicles to crowdsourced LAPs.

\subsection{Proposed Solution - Increasing Available Flight Power}

The power consumption on the autonomous unmanned aerial vehicle is sub-divided into four components. These are power expended by the (i) communication payload, (ii) sensing payload, (iii) motion payload and (iv) computing payload. The power consumed by the motion payload is responsible for the flight of the autonomous unmanned aerial vehicle. The communication payload enables data transmission between the LAP and the ground station. The sensing payload enables on-board camera operation and acquires training data. The computing payload consumes energy associated with using on-board trained neural networks to determine the flight configuration parameters and pre-processing of training data.

This paper proposes the use of neuromorphic computing hardware in the communication payload, sensing payload and computing payload. Neuromorphic computing hardware is suitable for future devices and technologies due to their low power consumption [31-32]. The use of neuromorphic hardware with low power consumption reduces the energy consumed by the communication payload, sensing payload and computing payload. This increases the proportion of the energy aboard the autonomous unmanned aerial vehicle that is available for the motion payload i.e. expended in flight.

The network scenario of the proposed mechanism is shown in Figure 1. The presented network scenario comprises the low altitude platform in the lower stratosphere, aerial vehicles in the class $\mathrm{G}$ airspace and ground based terrestrial wireless networks base stations. The altitude of the class $\mathrm{G}$ airspace and the low altitude platform relative to the ground location are $h_{2}$ and $h_{1}$ respectively. The distance between the class $\mathrm{G}$ airspace region and the low altitude platform is described by the relation $2 h_{2}>h_{1}$. Given that $2 h_{2}>h_{1}$, the use of the low altitude platform compared to terrestrial wireless network base stations results in a lower free space loss since $2 h_{2}>$ $h_{1}$. This reduces the energy consumption of the communication payload in the unmanned aerial vehicle.

The context in Figure 1 is one in which the low altitude platform based in the stratosphere has completed communications with the ground station. Hence, the ground station is not shown. However, low altitude platforms do not communicate continually with the ground stations but at scheduled intervals. The transmission of navigation control signals for non-autonomous unmanned aerial vehicles is not delivered timeously due to latency and network congestion. In Figure, 1, the low altitude platform is used to provide coverage and data transmissions to the concerned unmanned aerial vehicles. However, multiple low altitude platforms can be used for a larger coverage area. In this case, low altitude platforms provide coverage.

\subsection{Proposed Solution - Artificial Intelligence for Improved Airspace Safety}

The proposed mechanism aims to enhance airspace safety for autonomous unmanned aerial vehicles in the class $G$ airspace. The class $G$ airspace in the context of this paper can support remotely piloted aerial vehicles, optionally piloted aerial vehicles and autonomous unmanned aerial vehicles. The control of remotely piloted aerial vehicles and optionally piloted aerial vehicles from the ground stations via wireless networks is subject to delay and congestion in wireless networks. However, this is not true for autonomous unmanned aerial vehicles that use intelligent algorithms for flight guidance. 


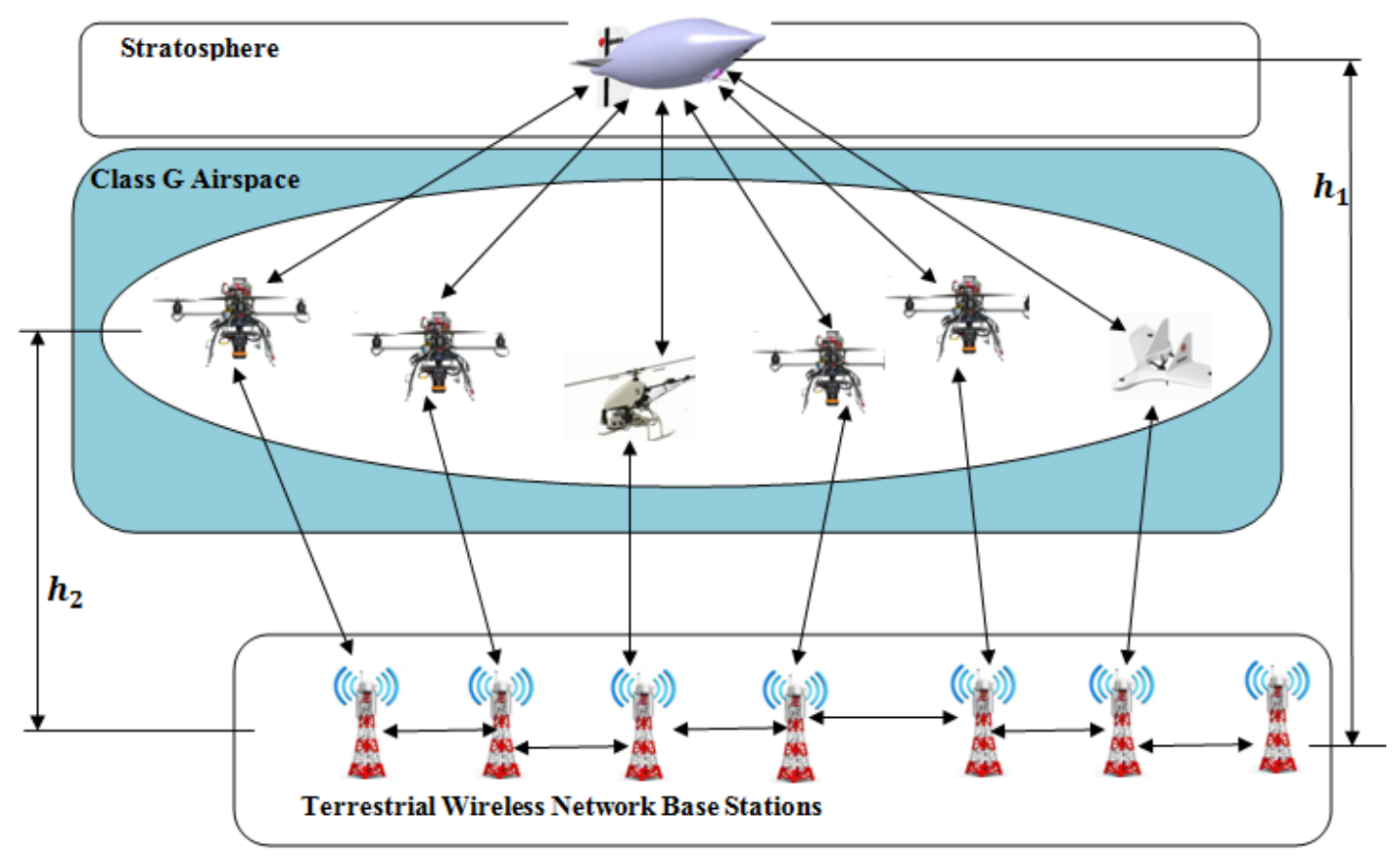

Figure 1: Proposed Mechanism's Network Scenario.

Training data required for intelligent algorithm training does not require continuous transmission.

The design of the proposed artificial intelligence paradigm is motivated by the intelligent pattern in which birds are observed to utilize the airspace. Birds demonstrate a high level of intelligence leading to the study of cognitive ornithology and avian intelligence [33-35]. Fox in [36] recognize that avian intelligence play an important role in the design of future multiintelligence systems. In [36], the combination of human and avian intelligence is used to design mechanisms enabling the design of useful techniques for drones. This combination requires the use of learning mechanisms motivated by human and avian intelligence.

Currently, artificial neural networks that play a significant role in artificial intelligence are motivated by the human brain. The role of avian intelligence in using the structures of bird brains to develop artificial neural networks is an area that remains largely unexplored. However, the joint use of artificial neural networks motivated by human and bird brains is essential for developing multi-intelligent hybrid learning systems. The goal of developing the multiintelligent hybrid learning systems can be achieved via learning diversity. Learning diversity is achieved by using artificial neural networks of different structures to realize the learning and prediction process. This is feasible because artificial neural networks have a significant number of configuration parameters.
Examples of configuration parameters for artificial neural networks are: (i) number of inputs, (ii) number of hidden layers, (iii) number of outputs, (iv) input layer transfer function, (v) pre-processing functions, (vi) Presence or absence of recurrent links, (vii) transfer function of each hidden layer, (viii) output layer transfer function, (ix) number of reservoirs (echo state network), (x) number of neurons in the input layer, (xi) number of neurons in each hidden layer and (xii) number of neurons in the output layer and (xiv) regularization parameter for each layer in the artificial neural network.

The values of these parameters can be varied and configured to be different thereby leading to the emergence of different artificial neural networks. The parameters influencing artificial neural network configuration are not limited to the aforementioned.

In the proposed mechanism, the LAP incorporates computing payload that hosts artificial neural network of different structures. The motivation for using multiple artificial neural networks is that the use of neural networks of different structures enables us to approximate the brain structure of birds. This paradigm is the approximate avian brain artificial neural network paradigm. The LAP acquired via open source and crowd-sourcing hosts payload comprising neuromorphic hardware. It develops training data obtained via observations made by the cameras mounted on autonomous unmanned aerial vehicles. 
The training data is developed via autonomous inference or user analysis via ground station access.

The training of the neural networks with different structures is done on the crowd-sourced low altitude platform. The training is done to predict configuration parameters given different environmental inputs as observed via the sensing payload. The output of the training procedure is the artificial neural network matrices. The developed matrices are transmitted to autonomous unmanned aerial vehicles that use them to determine flight configuration parameters.

The proposed artificial intelligence paradigm considers differences in camera capabilities in autonomous unmanned aerial vehicles. The possibility of using different sensing payload components implies that different artificial neural network structures will be suitable for different objectives. The crowd-sourced low altitude platform's computing payload executes multi-objective learning aiming to meet the learning demands and prediction objectives of autonomous unmanned aerial vehicles. The proposed solution also executes the task of learning algorithm classification. This enables the low altitude platform to determine the most suitable artificial neural network structure. Hence, parameter prediction in the aerial vehicle to be executed using the best learning mechanism at an epoch. The classification of learning mechanism is done by the learning mechanism classification entity aboard the LAP.

The multi-objective learning procedure incorporating learning diversity that is incorporated in the computing payload aboard the LAP is shown in Figure 2. Figure 2 shows the computing payload comprising neuromorphic computing hardware that pre-processes the acquired training data. In Figure 2, the depiction on the right shows the neuromorphic hardware hosting the implemented artificial neural networks. In addition, the LAP hosts computing facilities that provides storage for the matrices of the artificial neural network. The pre-processing of training data is done using multiple computing entities aboard the LAP. The pre-processed training data is used to train artificial neural networks and the output is a matrix for each neural network.

The sequence of relations between autonomous unmanned aerial vehicle and the LAP is shown in Figure 3. In Figure 3, the relations between the LAP (left) and the drone (right) occurs in four steps. In the first step, the drone transmits the data observed in the aerial environment to the LAP. The transmitted data is processed and used to develop training data aboard for the LAP. The processed data is used to train the artificial neural networks with different configurations that are hosted aboard the LAP. The output of the second step are well-trained artificial neural networks with own matrices. The third step involves the transmission of artificial neural network matrices from the LAP to autonomous unmanned aerial vehicle. Newly observed training data for a new scenario in the aerial environment is transmitted from the drone to the LAP in the fourth step.

\section{PERFORMANCE FORMULATION}

This section formulates the performance metrics of the proposed mechanism.

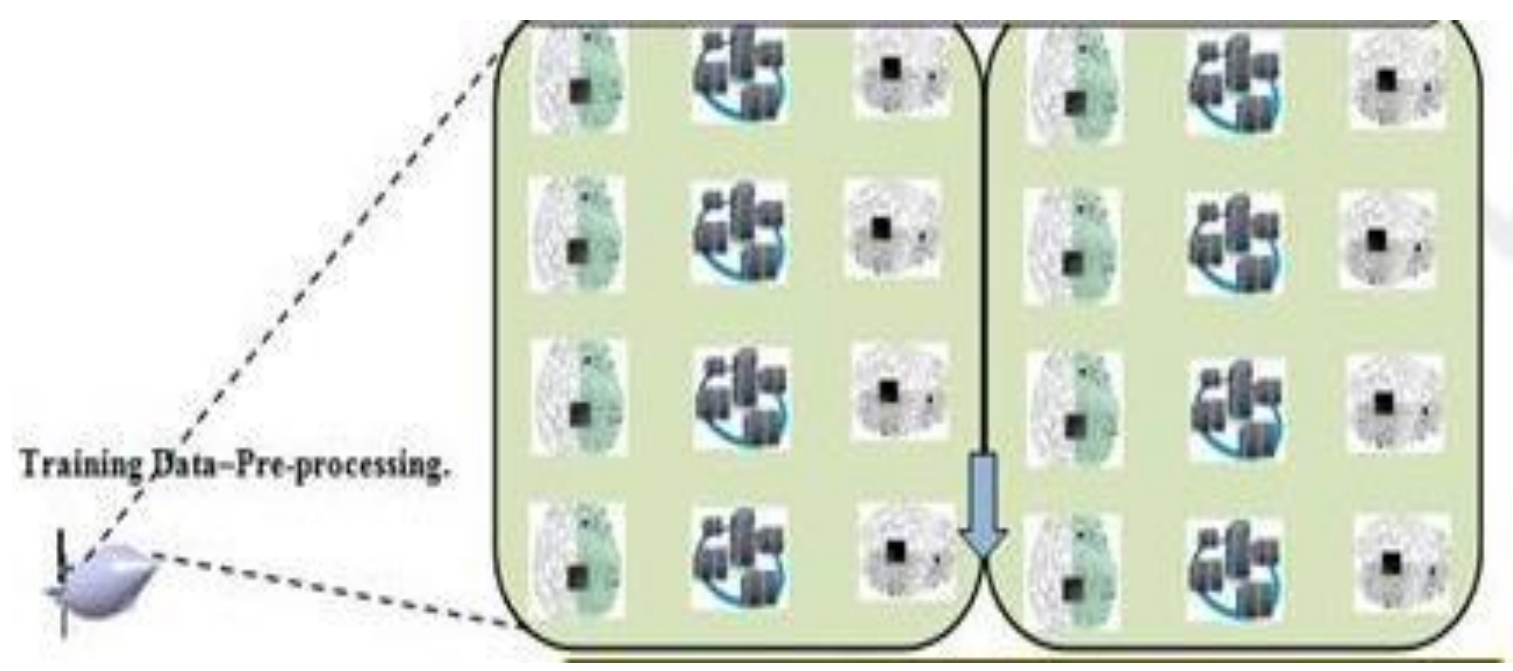

Figure 2: Machine learning aboard the low altitude platform in the proposed mechanism. 


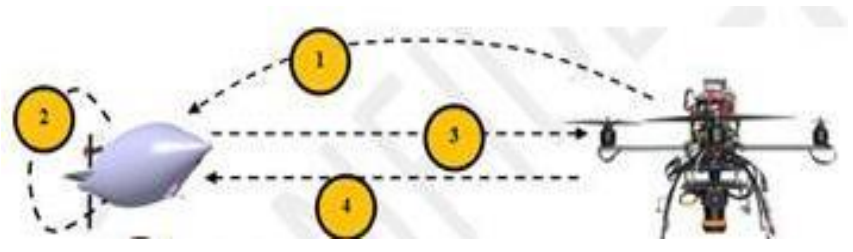

Figure 3: Relations between autonomous unmanned aerial vehicle and low altitude platform.

The formulated performance metrics are (i) wireless network access costs, (ii) available flight power onboard autonomous unmanned aerial vehicle and (iii) aerial aviation safety index (AASI). This section is divided into three parts. The first part focuses on the wireless network access costs. The second part formulates the available flight power on-board the autonomous aerial vehicle and available flight range. The third part focuses on the AASI.

\subsection{Performance Formulation - Wireless Network Access Costs}

The wireless network access costs when terrestrial wireless networks base stations are utilized to transmit information on developed artificial neural network matrices to autonomous unmanned aerial vehicles is denoted $Y_{1}$. The network access costs when low altitude platforms are required to transmit information on developed artificial neural network matrices is denoted $Y_{0}$. The costs $Y_{0}$ and $Y_{1}$ are formulated considering the normalized load due to aerial vehicle traffic and that due to terrestrial network denoted as $\rho_{u}\left(\alpha_{i}^{j}, t_{y}\right)$ and $\rho_{t r_{-} b s}\left(\alpha_{i}^{j}, t_{y}\right)$ respectively.

$Y_{n}=\sum_{y=1}^{Y} \sum_{j=1}^{J} P_{a c}\left(\alpha_{i}^{j}, t_{y}\right)\left(C_{d}^{1}\left(\alpha_{i}^{j} t_{y}\right)+\right.$ $\left.C_{d}^{2}\left(\alpha_{i}^{j}, t_{y}\right)\right) \exp \left(\rho_{u}\left(\alpha_{i}^{j}, t_{y}\right)+n \rho_{t r_{b s}}\left(\alpha_{i}^{j}, t_{y}\right)\right)+$ $\left.\frac{\vartheta^{\prime}}{|\alpha|}\right|_{n=0}$

$\epsilon\{0,1\}$

Where $\vartheta^{\prime}$ is the cost of acquiring or developing the low altitude platform via open source strategy.

\subsection{Performance Formulation - Available Flight Power}

The available flight power is formulated for the case where the autonomous unmanned aerial vehicle communicates with either the terrestrial wireless network base station or low altitude platform. The data transmit power when the autonomous unmanned aerial vehicle transmits data to the terrestrial wireless network depends on the altitude and frequency.

Let $P_{t r_{-} b s}\left(\alpha_{i}^{j}, t_{y}, h_{2}, f\right)$ denote the transmit power required by autonomous unmanned aerial vehicle $\alpha_{i}^{j}$ to transmit to the base station from an altitude $h_{2}$ at frequency $f$ at epoch $t_{y}$. In addition, $P_{t r \_h a}\left(\alpha_{i}^{j}, t_{y},\left(h_{1}-h_{2}\right), f\right)$ is the transmit power required by autonomous unmanned aerial vehicle $\alpha_{i}^{j}$ at altitude $\left(h_{1}-h_{2}\right)$ and frequency $f$ at epoch $t_{y}$. The power consumed by the communication payload in the autonomous unmanned aerial vehicle comprises power expended in pre-processing data for data transmission.

The parameters $P_{t r_{-} h a}\left(\alpha_{i}^{j}, t_{y},\left(h_{1}-h_{2}\right), f\right)$ and $P_{t r_{-} b s}\left(\alpha_{i}^{j}, t_{y}, h_{2}, f\right)$ are associated with the free space loss given that autonomous unmanned aerial vehicles are not deployed in rainfall. The formulation assumes that the sensing payload consumes the same amount of power in both cases. The available flight power associated with $P_{t r_{-} h a}\left(\alpha_{i}^{j}, t_{y},\left(h_{1}-h_{2}\right), f\right)$ and $P_{t r \_b s}\left(\alpha_{i}^{j}, t_{y},\left(h_{1}-h_{2}\right), f\right)$ are denoted as $P_{f l_{\_} h a}\left(h_{1}-\right.$ $\left.h_{2}, f\right)$ and $P_{f l_{-} b s}\left(h_{2}, f\right)$ respectively.

$$
\begin{gathered}
P_{f l_{-} b s}\left(\alpha_{i}^{j}, h_{2}, f\right)=P-\left(\sum_{y=1}^{Y} P_{t r_{-} b s}\left(\alpha_{i}^{j}, t_{y}, h_{2}, f\right)\right)(8) \\
P_{f l_{\_} h a}\left(\alpha_{i}^{j},\left(h_{1}-h_{2}\right), f\right)=P-\left(\sum _ { y = 1 } ^ { Y } P _ { t r _ { h a } } \left(\alpha_{i}^{j}, t_{y},\left(h_{1}-\right.\right.\right. \\
\left.\left.\left.h_{2}\right), f\right)\right)
\end{gathered}
$$

\subsection{Performance Formulation - Influence of Artificial Intelligence Algorithm on Air Safety}

The proposed mechanism advocates the incorporation of multi-intelligence paradigm i.e. learning diversity airspace safety when autonomous unmanned aerial vehicles are deployed.

The influence of artificial intelligence on enhancing air safety is formulated via the AASI metric. The AASI gives a measure of aerial safety considering the performance of artificial neural networks used in autonomous unmanned aerial vehicles. Artificial neural networks can be used to predict different configuration parameters such as speed, flight direction, hovering duration and necessary altitude adjustments amongst other parameters.

These outputs can be determined with varying degrees of prediction accuracy for an artificial neural network. Hence, the prediction accuracy is an 
important parameter for the artificial neural network or any other learning mechanism.

Let ' $\Upsilon$ denote the set of artificial neural networks that can be used by autonomous unmanned aerial vehicles such that:

$$
\text { ' } \Upsilon=\left\{\Upsilon_{1}, \Upsilon_{2}, \ldots, \Upsilon_{M}\right\}
$$

Where $M$ is the total number of artificial neural networks.

The probability that the prediction accuracy of the artificial neural network ' $\Upsilon_{m},{ }^{\prime} \Upsilon_{m} \in$ ' $\Upsilon$ at epoch $t_{y}$ does not meet or exceed the desired predicted output is denoted $P\left({ }^{\prime} \Upsilon_{m}, t_{y}\right)$.

Furthermore, the artificial neural networks are classified based on their outputs i.e. prediction accuracy. This functionality is executed by the learning mechanism classification entity. Let $\ddot{Y}$ denote the set of learning mechanism classification entities such that:

$$
\ddot{\Upsilon}=\left\{\ddot{\Upsilon}_{1}, \ddot{\Upsilon}_{2}, \ldots, \ddot{\Upsilon}_{N}\right\}
$$

Where $N$ is the total number of learning mechanism classification entities.

In the existing mechanism i.e. [37], the autonomous unmanned aerial vehicle incorporates one artificial neural network structure such as the convolutional neural network. The airspace safety is compromised if the artificial neural network prediction falls short or exceeds the value of the desired output. The AASI assuming that the autonomous aerial unmanned vehicle incorporates artificial neural networks in an ensemble is denoted $\Gamma_{1}$. In this case, the artificial neural networks or other learning algorithms are not classified.

$$
\Gamma_{1}=\left(1-\left(\prod_{m=1}^{M} \prod_{y=1}^{Y} P\left(\Upsilon_{m}, t_{y}\right)\right)\right)
$$

In the proposed mechanism, learning algorithm classification is executed by the learning algorithm classification entity. The probability that the learning algorithm classification entity $\ddot{\Upsilon}_{n}, \ddot{\Upsilon}_{n} \in \ddot{Y}$ does not function as expected at epoch $t_{y}$ is denoted $P\left(\ddot{\Upsilon}_{n}, t_{y}\right)$. The airspace safety is compromised if all the artificial neural networks suitable for prediction in a given scenario fail to deliver expected performance or if the learning mechanism classification entities fail to deliver the expected performance. The AASI when the autonomous aerial unmanned vehicle incorporates learning diversity is denoted $\Gamma_{2}$ and given as:

$$
\begin{aligned}
& \Gamma_{2}=\left(1-\left(\prod_{m=1}^{M} \prod_{y=1}^{Y} P\left(\Upsilon_{m}, t_{y}\right)\right)\right)+(1- \\
& \left.\left(\prod_{n=1}^{N} \prod_{y=1}^{Y} P\left(\ddot{\Upsilon}_{n}, t_{y}\right)\right)\right)
\end{aligned}
$$

\section{PERFORMANCE SIMULATION}

This section presents the result of performance simulation and discusses the performance benefits of using the proposed mechanism. The performance simulation, evaluation and analysis are done using the MATLAB software package. The MATLAB software package has been used because it has a flexible editor tool that can allow the evaluation of the proposed mechanism under different performance conditions and scenarios. The use of the editor tool in MATLAB for the performance simulation and evaluation requires the provision of parameters describing the scenario where the proposed mechanism is deployed. The parameters that are used in this regard are presented in Table I.

The performance simulation has also assumed that unmanned aerial vehicles can have different altitudes, normalized traffic load, size of control data and training data and probability of failure for learning mechanisms. In addition, the mean values can be easily obtained for random values that can be defined using MATLAB inbuilt functions such as the 'randtool'. This has necessitated the providing the information on the average value of these parameters in the information presented in Table 1.

The performance simulation is done using the parameters shown in Table I. The simulation is done with the aim of investigating how the use of the proposed mechanism influences the network access costs, the available flight power and the AASI.

The result for the network access costs is shown in Figure 4 and Figure 5. Figure 4 and Figure 5 shows network access costs for the proposed mechanism and existing approach respectively. The network access cost for the existing approach in is examined for the case where the normalized traffic load for wireless subscribers has values of $1.5,3.0$ and 4.5 as described in Case 1, Case 2 and Case 3 respectively.

The scenario describing the existing case can be found in [27] where mobile networks such as the LTEAdvanced and $5 \mathrm{G}$ networks are used to transmit all data associated with drone control. The result in Figure 4 are for the case of the proposed mechanism low altitude platform is used to execute only data transmission related to unmanned aerial vehicle. The network access costs in Figure 5 have been determined using the scenario in [27] as the case study. In the simulation, three cases of normalized load have been considered. 
Analysis of the results presented in Figure 4 and Figure 5 show that the use of the proposed mechanism reduces network access costs in comparison to results obtained from existing mechanism. The proposed mechanism reduces the network access costs by an overall mean of $90 \%$. Therefore, the use of the proposed mechanism in managing autonomous unmanned aerial traffic instead of using terrestrial wireless network base station is beneficial. This is because of the significant improvement even when terrestrial wireless network has low congestion levels i.e. low normalized traffic load level.

The results on the available flight power on-board the autonomous unmanned aerial vehicle is shown in Figure 6. In Figure 6, the existing approach refers to the existing scenario in [28] where unmanned aerial vehicles are controlled from the terrestrial mobile network. The proposed approach refers to the scenario in the proposed mechanism.

The results in Figure 6 show that the available flight power increases with unmanned aerial vehicle altitude. This is because the autonomous unmanned aerial vehicle is closer to the low altitude platforms as its altitude increases. This reduces the influence of free space loss in the case of the proposed mechanism. Therefore, the communication payload uses less proportion of the energy to overcome free space loss and realize intended communications. Hence, more energy is available for flight.

However, in the existing approach [27], separation between the autonomous unmanned aerial vehicle and the terrestrial wireless network base station increases with aerial vehicle altitude. Therefore, the available flight power decreases with increasing altitude in the existing scheme.

This is because a larger proportion of the power in the autonomous unmanned aerial vehicle is expended to overcome the higher free space path loss.

Analysis of the results in Figure 6 shows that the use of the proposed mechanism increases aerial vehicle flight power by $37.3 \%$ on average. An increment in the power available for flight increases aerial vehicle range and increases power available to operate more components that enhances safety.

The simulation result for the AASI is shown in Figure 7. The AASI is examined for three scenarios i.e. Scenario 1, Scenario 2 and Scenario 3.

Scenario 1 describes the case for which artificial neural networks are used to predict the configuration parameter of the autonomous unmanned aerial vehicle. This describes the existing scenario that can be found in [37]. The discussion in [37] utilizes a welltrained convolutional neural network for navigation in autonomous unmanned aerial vehicles.

The convolutional neural network generates the steering commands for the unmanned aerial vehicle. It is assumed that the convolutional neural network used in [37] can predict the required steering commands for all scenarios with $100 \%$ accuracy. It does not consider scenarios for which the change in the configuration of the parameters of the convolution neural network might be necessary.

Scenario 2 and Scenario 3 describes the case where the proposed multi-intelligence framework incorporating learning diversity is incorporated. This is the case with the existing mechanism. The difference between scenarios 2 and 3 is the number of learning algorithm classification entities.

Table 1: Simulation Parameters

\begin{tabular}{lll}
\hline S/N & Parameter & Value \\
\hline 1 & Low Altitude Platform - Altitude & $10 \mathrm{~km}$ \\
2 & Mean altitude of autonomous unmanned aerial vehicle & $6.7 \mathrm{~km}$ \\
3 & Autonomous unmanned aerial vehicle on-board power & $1 \mathrm{MW}$ \\
4 & Operational Frequency & $2 \mathrm{GHz}$. \\
5 & Mean Normalized Traffic Load of autonomous unmanned aerial vehicle & 12 \\
6 & Normalized Traffic Load of subscriber traffic & {$\left[\begin{array}{ll}0.8 \quad 1.6 \quad 2.4 \\
7\end{array}\right.$} \\
8 & Mean size of transmitted controlled data & $477.6 \mathrm{Bytes}$ \\
9 & Mean size of transmitted training data & $0.525 \mathrm{Mbytes}$ \\
10 & Mean network access costs per MB. (conservative estimate) & $\$ 7.5$ \\
11 & Mean probability of failure of artificial neural network & 10 \\
12 & Overall number of learning mechanism classification entities & 0.6148 \\
13 & Mean probability of failure of learning mechanism classification entities & 3 \\
\hline
\end{tabular}


The number of classification entities in scenario 2 and scenario 3 is one and three respectively.

Analysis shows that the AASI in scenario 2 and scenario 3 outperforms that of scenario 1 by an average of $29.7 \%$ and $53.2 \%$ respectively. An increase in the number of learning mechanism classification entities from 1 to 3 by $33.3 \%$ enhances the AASI by an average of $35.3 \%$.

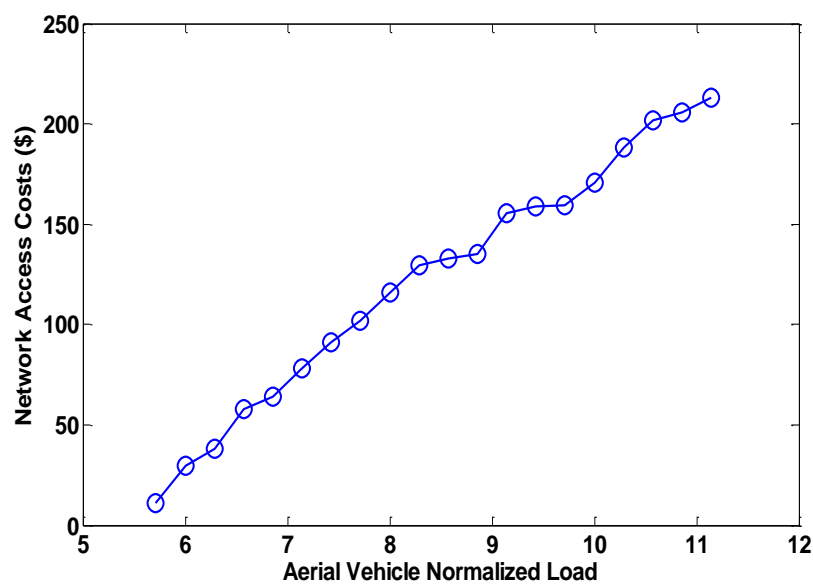

Figure 4: Network Access Costs in the proposed mechanism.

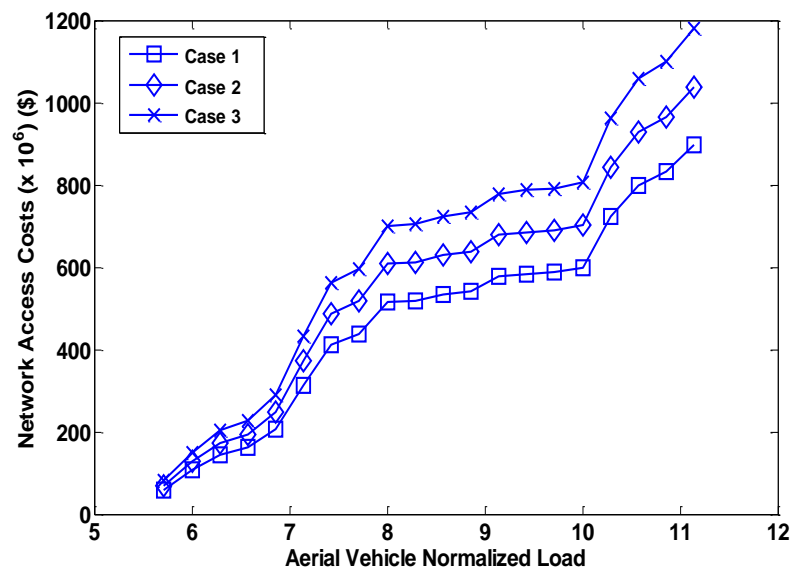

Figure 5: Network Access Costs in the existing mechanism.

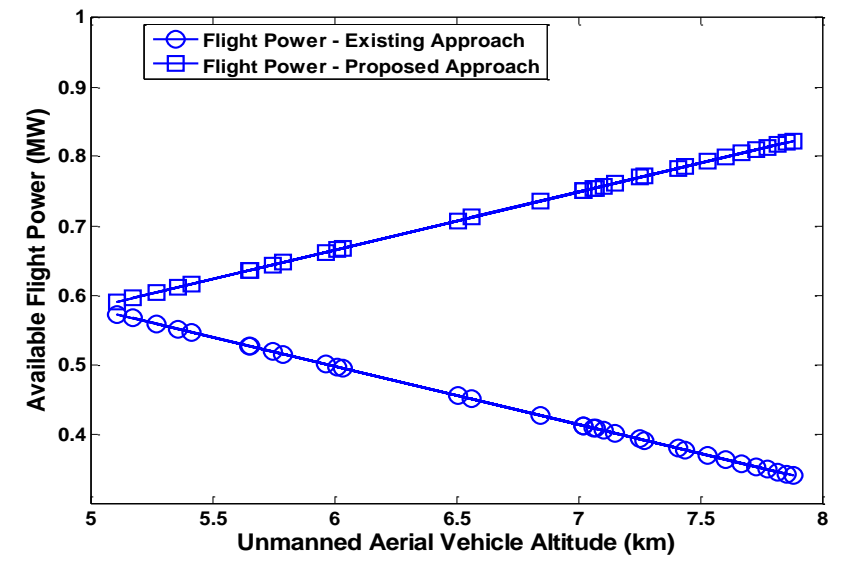

Figure 6: Simulation results for the available flight power.

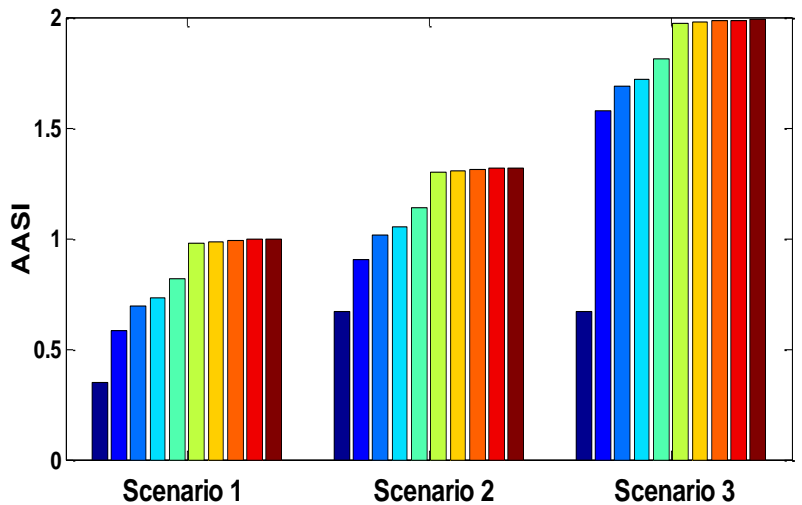

Figure 7: Simulation results for the AASI.

\section{CONCLUSION}

The discussion in this paper addressed the challenge of ensuring airspace safety when a significant number of unmanned aerial vehicles are deployed in civil applications. The paper proposed the use of low altitude platforms hosting intelligent algorithms and learning diversity to improve the safety of deployed unmanned aerial vehicles. It has also identified and described the relations between different entities that interact to ensure that the goal of airspace safety is realized. The paper also formulated the performance metrics used to examine the performance of the proposed mechanism. The performance benefit of the proposed mechanism is evaluated and analyzed by using the MATLAB software package. The results obtained show that the use of the proposed mechanism outperformed the existing approach where terrestrial wireless network base stations are used for unmanned aerial vehicle control. The available flight power and air safety are improved by an average of $37.3 \%$ and $53.2 \%$ respectively when the proposed mechanism is used.

\section{REFERENCES}

[1] D. Palma, A. Zolich, Y. Jiang and T.A. Johansen, 'Unmanned Aerial Vehicles as Data Mules: An Experimental Assessment', IEEE Access, Vol. 5, pp 24716 - 24726, 2017.

[2] W.Shi, H.Zhou, J.Li, W.Xu, N. Zhang, and X.Shen, 'Drone Assisted Vehicular Networks: Architecture, Challenges and Opportunities', IEEE Network, Vol. 32, No. 3, pp 130 - 137, 2018.

[3] V.Sharma, K.Srinivasan, H. Chao, K.Hua, and W.Cheng, 'Intelligent Deployment of UAVs in 5G Heterogeneous Communications Environment for Improved Coverage', Journal of Networks and Computer Applications, Vol. 85, Issue. C, pp 94105, May 2017. 
[4] E.Moguel, J.M.Conejero, F.Figuerva, J.Hernandez, J.C.Preciado and R.Echeverria, 'Towards the use of unmanned aerial systems for providing sustainable services in smart cities', Sensors (Basel), Vol. 8, No. 64, pp 1-24, 27 Dec 2017.

[5] J.Jacob, D.Axisa, and S.Onchey, 'Unmanned Aerial Systems for Atmospheric Research: Instrumentation Issues for Atmospheric Measurements', [Online] https://www.eol.ucar.edu/system/files/instrume ntation\%20\%Paper.pdf Feb, 14, 2017, Accessed $07 / 10 / 2018$.

[6] T.Niedzielski, C.Skjoth, M.Werner, W.Spallek, M.Witek, T.Sawinski, A.D.Osiadacz, M.Muskada, P.Muskala, P.Modzal, J.Guzskowski, and M.Kryza, 'Are Estimates of wind characteristics based measurements with Pitot tubes and GNSS receivers mounted on consumer grade unmanned aerial vehicles application in meteorological studies', Environmental Monitoring and Assessments, Vol. 189, No. 431, pp 1 - 18, 2017.

[7] A.M.Picar, C.Marque, M.Anciauz, H.Larry and S.Ranvier, 'Antenna Pattern Calibration of radio telescopes using an UAV based device', International Conference on Electromagnetics in Advanced Applications, Turin, Italy, pp 981 984, , 7 - 11 Sept, 2015.

[8] A.G.Basden, A.M.Brown, P.M.Chadwick, P.Clark, and R.Massey, 'Artificial guide stars for adaptive optics using unmanned aerial vehicles', Monthly Notices of the Royal Astronomical Society, Vol. 477, No. 2, pp 2209 - 2219, 21 June 2018.

[9]A.A Periola and O.E. Falowo, 'Instrumentation Location Diversity Paradigm for Future Astronomy Observations', Wireless Personal Communications, Vol. 103, No. 3, pp 2475 2499, Sept 2018.

[10] C.Stocker, R.Bennett, F.Nex, M.Gerke and J.Zevenberger, 'Review of the current state of UAV regulations', Remote Sensing, Vol. 9, No. 5, pp $1-26,2017$.

[11] V.Chang, P.Chundury, and M.Chetty, 'Spiders in the Sky : User Perceptions of Drones, Privacy and Security', Proceedings of the 2017 CHI Conference on Human factors in computing systems, Denver, Colorado, pp 6765 - 6776, May $06-11,2017$.

[12] G.Choudhary, V.Sharma, T.Gupta, J.Kim and I.You, 'Internet of Drones (IoD): Threats, Vulnerability and Security Perspectives', Symposium on Mobile Internet Security (MobiSec '18), Cebu, Phillipines, Article No. 37, pp $1-13$, Aug 29 - Sep 1, 2018.
[13] M.Lyons, K.Brandis, C.Callaghan, J.McCann, C.Mills, S.Ryall and R.Kingsford, 'Bird Interaction with drones from individuals to large colonies', Australian Field Ornithology, 35, pp 51-56, 2018.

[14] H.Shakhatrah, A.Sawelmeh, A.Fuqala, Z.Dou, E.Almaita, I.Khalil, N.S.Othman, A.Khreishah and M.Guizani, 'Unmanned Aerial Vehicles: A Survey on civil applications and key research challenges', IEEE Access , Vol. 7, pp 48572 - 48634, 09 April 2019.

[15]S.Hayat, E.Yanmaz and R.Muzaffar, 'Survey on unmanned aerial civil applications: A communications viewpoint', IEEE Communications Surveys \& Tutorials, Vol. 18, No. 4, pp 2624-2661, 2018.

[16] R.T.Ogan, 'Integration of manned and unmanned aircraft systems into U.S. airspace', IEEE SOUTHEASTCON, Lexington, KY, USA, pp $1-4$, 13 - 16 March 2014.

[17] A. Carrio, C.Sampedro, A.Ramos, and P.Campoy, 'A Review of Deep Learning Methods and Application for Unmanned Aerial Vehicles', Journal of Sensors, Hindawi, Vol. 2017, Article ID 3296874, pp 1-13, 2017

[18] A. Kouris and C.Bouganis, 'Learning to Fly by MySelf: A Self-Supervised CNN-based Approach for Autonomous Navigation', IEEE/RSJ International Conference on Intelligent Robots and Systems (IROS), Madrid Spain, pp 1-9, 1-5 Oct 2018.

[19] C.Luo, W.Zhao, Z.Du and L.Yu, 'A Neural Network Based Landing Method for an Unmanned Aerial Vehicle with Soft Landing Gears', Applied Sciences, 9(15), 2976, pp 1 - 16, 2019.

[20]W.Shyy, C.Kang, P.Chirarattanaron, S.Ravi and H.Liu, 'Aerodynamics, sensing and control of insect-scale flapping-wing flight', Proc. $R$. Soc. A 472: 20150712, pp 1-37, 2016.

[21] C.F.Liew, D.DeLatte, N.Takeishi and T.Yairi, 'Recent Developments in Aerial Robotics: A Survey and Prototypes overview', arXiv, [Online] Available https://arxiv.org/pdf/1711.10085.pdf, pp 1-14, 2017.

[22]R.Yudhono and A.A. Erissonia, 'Design of a Birdlike Fixed-wing Unmanned Aerial Vehicle', International Conference on Engineering and Natural Science-Summer Session, Tokyo, Japan, pp 65 -72, July 30 - August 1, 2018.

[23]E.Ackerman, 'Artificial Feathers Let Drones Morph Their Wings Like Birds', IEEE Spectrum, [Online] Available: https://spectrum.ieee.org/automaton/robotics/d 
rones/artificial-feathers-let-drones-morph-theirwings-like-birds, 20 Dec 2016

[24]M.D.Luca, S.Mintchev, G.Heitz, F.Noca and D.Floreano, 'Bio-inspired morphing wings for extended flight envelope and roll control of small drones' Interface Focus Vol.7, No.1, pp 1-11, 2016.

[25]F.T.Puthri, M.Ariyanto, I.Haryanto, M.Arozi, W.Caesarenda, and M.R.I.Hanan, 'Development of Unmanned Aerial Vehicle (UAV) ornithopter with wireless radio control', International Conference on Information Technology, Computer, and Electrical Engineering (ICITACEE), Semarang, Indonesia, pp $79-84$, 19-20 Oct 2016

[26] A.A Periola and O.E. Falowo, 'A robust conscious model for enhancing cognitive radio quality of service', IEEE Annual International Symposium on Personal, Indoor, and Mobile Radio Communications (PIMRC), Valencia, Spain, pp 16, 4-8 Sept 2016.

[27]G.Yang, X.Lin, Y.Li, H.Cui, M.Xu, D.Wu, H.Ryden and S.Redhwan, 'A Telecom Perspective on the Internet of Drones from LTE-Advanced to $5 \mathrm{G}^{\prime}$, arXiv [Online] Available: https://arxiv.org/ftp/arxiv/papers/1803/1803.11 048.pdf, pp 1-8, 2018.

[28]Ericsson, 'Drones and networks: Ensuring safe and secure operations', GFMC-18:00526, [Online] Available: https://www.ericsson.com/4adfc0/assets/local/p ublications/whitepapers/10201110 wp dronesandmobilenetwork s nov18.pdf, 2018.

[29]D.Colas, N.Roberts and V.Samuels, 'Opensourcing Facebook's Solar-Powered Aircraft Design Tools', Connectivity, Open Source, Facebook, facebook Engineering, [Online] Available: https://engineering.fb.com/connectivity/opensourcing-facebooks-solar-powered-aircraftdesign-tools/, Aug 17, 2018.

[30]H.Hall, B.Donitz, L.Kim, D.Srivastava, K.Albee, S.Eisner, D.Pierce, Y.Villapudua and A.Stoica, 'Project Zephyrus: Developing a Rapidly Reusable High-Altitude Flight Test Platform', IEEE Aerospace Conference, Big Sky, MT, USA, pp 137, 3-10 March 2018.

[31] N.K. Upadhyay, H.Jiang, Z. Wang, S.Asapu, Q.Xia, and J.J.Yang', Emerging Memory Devices for Neuromorphic Computing', Adv. Mater. Technol. 4, 1800589, pp 1-13, 2019.

[32]F. Hadaeghi, 'Neuromorphic Electronic Systems for Reservoir Computing', 2019, arXiv, [Online] Available-https://arxiv.org/pdf/1908.09572.pdf, pp $1-17,2019$.

[33] Z.A Zorina and T.A. Obozova, 'New Data on the Brain and Cognitive Abilities of Birds', Biology Bulletin, Vol. 39, No. 7, pp 601-617, 2012.

[34] T.Nomura and E. Izawa, 'Avian brains: Insights from development, behaviours and evolution', Development, Growth and Differentiation, Vol. 59, pp $244-257,2017$.

[35] N.J. Emery, 'Cognitive ornithology: the evolution of avian intelligence', Philos Trans $R$ Soc Lond $B$ Biol Sci. , 361(1465): 23-43, 29 Jan 2006.

[36] S.Fox, 'Beyond AI: Multi-Intelligence (MI) Combining Natural and Artificial Intelligences in Hybrid Beings and Systems', Technologies, Vol. 5, No. 3, 38, pp 1 - 14, 2017.

[37]K.Amer, M.Samy, M.Shaker and M.Elhelw,' Deep Convolutional Neural Network-Based Autonomous Drone Navigation', arXiv, [Online] Available:

https://arxiv.org/ftp/arxiv/papers/1905/1905.01 657.pdf, pp 1-8, 2019. 